\title{
OPEN The anti-inflammatory effects of Akkermansia muciniphila and its derivates in HFD/CCL4-induced murine model of liver injury
}

\author{
Shahrbanoo Keshavarz Azizi Raftar ${ }^{1,2,3,7}$, Fatemeh Ashrafian ${ }^{1,3,4,7}$, Sara Abdollahiyan ${ }^{2}$, \\ Abbas Yadegar ${ }^{2}$, Hamid Reza Moradi ${ }^{5}$, Morteza Masoumi ${ }^{3}$, Farzam Vaziri ${ }^{1,3}$, Arfa Moshiri ${ }^{1,3}$, \\ Seyed Davar Siadat ${ }^{1}{ }^{1,3 凶}$ \& Mohammad Reza Zali ${ }^{6 \bowtie}$
}

Inflammation plays a critical role in the promotion of hepatocyte damage and liver fibrosis. In recent years the protective role of Akkermansia muciniphila, a next-generation beneficial microbe, has been suggested for metabolic and inflammatory disorders. In this study, we aimed to evaluate the effects of live and pasteurized $A$. muciniphila and its extra cellular vesicles (EVs) on inflammatory markers involved in liver fibrosis in a mouse model of a high-fat diet (HFD)/carbon tetrachloride $\left(\mathrm{CCl}_{4}\right.$ )-induced liver injury. Firstly, the responses of hepatic stellate cells (HSCs) to live and pasteurized $A$. muciniphila and its EVs were examined in the quiescent and LPS-activated LX-2 cells. Next, the anti-inflammatory effects of different forms of $A$. muciniphila were examined in the mouse model of $\mathrm{HFD} / \mathrm{CCl}_{4}$-induced liver injury. The gene expression of various inflammatory markers was evaluated in liver, colon, and white adipose tissues. The cytokine secretion in the liver and white adipose tissues was also measured by ELISA. The results showed that administration of live and pasteurized $A$. muciniphila and its EVs leads to amelioration in HSCs activation. Based on data obtained from the histopathological analysis, an improvement in gut health was observed through enhancing the epithelium and mucosal layer thickness and strengthening the intestinal integrity in all treatments. Moreover, live A. muciniphila and its EVs had inhibitory effects on liver inflammation and hepatocytes damage. In addition, the tissue cytokine production and inflammatory gene expression levels revealed that live $A$. muciniphila and its EVs had more pronounced anti-inflammatory effects on liver and adipose tissues. Furthermore, EVs had better effects on the modulation of gene expression related to TLRs, PPARs, and immune response in the liver. In conclusion, the present results showed that oral administration of $A$. muciniphila and its derivatives for four weeks could enhance the intestinal integrity and antiinflammatory responses of the colon, adipose, and liver tissues and subsequently prevent liver injury in $\mathrm{HFD} / \mathrm{CCL}_{4}$ mice.

Liver disease is the cause of $2-4 \%$ of deaths annually worldwide. It encompasses a wide range of clinical conditions, including nonalcoholic fatty liver disease (NAFLD), nonalcoholic steatohepatitis (NASH), alcoholic liver disease (ALD), and viral hepatitis ${ }^{1,2}$. NAFLD is the most common liver disease with a global prevalence of $25 \%$. It is the main cause of advanced liver fibrosis, hepatocellular carcinoma (HCC), and liver failure ${ }^{3}$. A normal liver tissue consists of 5-10\% quiescent hepatic stellate cells (HSCs), which are non-parenchymal cells located in the perisinusoidal space of the liver ${ }^{4}$. Activated HSCs are characterized by the expression of fibrosis markers and excessive accumulation of extracellular matrix (ECM) proteins, contributing to the onset and progression

\footnotetext{
${ }^{1}$ Microbiology Research Center (MRC), Pasteur Institute of Iran, Tehran, Iran. ${ }^{2}$ Foodborne and Waterborne Diseases Research Center, Research Institute for Gastroenterology and Liver Diseases, Shahid Beheshti University of Medical Sciences, Tehran, Iran. ${ }^{3}$ Department of Mycobacteriology and Pulmonary Research, Pasteur Institute of Iran, Tehran, Iran. ${ }^{4}$ Clinical Research Departments, Pasteur Institute of Iran, Tehran, Iran. ${ }^{5}$ Department of Basic Sciences, School of Veterinary Medicine, Shiraz University, Shiraz, Iran. ${ }^{6}$ Gastroenterology and Liver Diseases Research Center, Research Institute for Gastroenterology and Liver Diseases, Shahid Beheshti University of Medical Sciences, Tehran, Iran. ${ }^{7}$ These authors contributed equally: Shahrbanoo Keshavarz Azizi Raftar and Fatemeh Ashrafian ${ }^{\square}$ email: d.siadat@gmail.com; nnzali@hotmail.com
} 
of liver fibrosis. Inflammation plays an important role in triggering the activation of HSCs through cytokine secretion by the adjacent Kupffer cells ${ }^{5}$.

A large number of microorganisms inhabit the human gastrointestinal (GI) tract, affecting the host's health status through maintaining metabolic and immune homeostasis and protecting against pathogens ${ }^{6}$. There is a bilateral connection between the gut and the liver, known as the gut-liver axis; accordingly, the gut microbial products and metabolites are directly conveyed across the portal vein into the liver ${ }^{7}$. Recent evidence shows that changes in the composition of the gut microbiota are associated with many pathological conditions in various liver diseases ${ }^{8}$. Therefore, modulation of the gut microbiota composition can be a potential approach for preventing liver damage?

Akkermansia muciniphila (A. muciniphila) is an aerotolerant anaerobic mucin-degrading bacterium that inhabits the human GI tract ${ }^{10,11}$. Studies have disclosed the protective and regulatory role of $A$. muciniphila as a next-generation beneficial microbe in the metabolic and immune functions of mice that were fed a high-fat $\operatorname{diet}(\mathrm{HFD})^{12-15}$. A series of human and animal studies also support the efficacy of A. muciniphila supplementation as a new therapeutic option for the management of obesity and obesity-related metabolic disorders ${ }^{16,17}$. The pasteurized form of $A$. muciniphila can also increase protection against metabolic disorders in HFD-fed mice through increasing the number of goblet cells, which in turn increases the thickness of mucosal layer and improves the intestinal barrier function ${ }^{14}$.

Extracellular vesicles (EVs) are membrane-enclosed vesicles, produced by all types of organisms that play vital roles in intercellular communication and many physiological and pathological processes ${ }^{18-20}$. A variety of medical applications, including vaccine development, adjuvant therapy, delivery systems, and diagnostic and therapeutic applications, have been described for $\mathrm{EVs}^{21}$. Besides, EVs derived from the intestinal microbiota can be transmitted to various organs through penetration into the circulatory system and lead to a wide range of immunological responses ${ }^{22,23}$. Evidence suggests that EVs derived from the intestinal microbiota can establish an effective cross-talk between the microbiota and the host immune response, which may be related to the components on the surface of $\mathrm{EVs}^{24}$.

In 2019, we found that A. muciniphila and its EVs had anti-obesity effects on HFD-fed mice by increasing the mRNA level of peroxisome proliferator-activated receptor (PPAR) genes in white adipocytes and inducing immune hemostasis in the colon tissue ${ }^{13}$. Moreover, in 2021, we demonstrated that heat-killed A. muciniphila could inhibit HSC activation and induce HSC regression in the lipopolysaccharide (LPS)-activated LX-2 cell line ${ }^{25}$.Accordingly, in the present study, we aimed to evaluate the effects of live and pasteurized A. muciniphila and its EVs on inflammatory markers involved in liver fibrosis in a mouse model of HFD/carbon tetrachloride $\left(\mathrm{CCl}_{4}\right)$-induced liver injury.

In this study, the responses of HSCs to live and pasteurized A. muciniphila and its EVs were examined in the quiescent and LPS-activated LX-2 cell line. Next, the anti-inflammatory effects of different forms of A. muciniphila and its EVs were examined in the liver tissue of a mouse model of $\mathrm{HFD} / \mathrm{CCl}_{4}$-induced liver injury, and the gene expression of various inflammatory markers was evaluated in the mouse liver tissue. Moreover, the expression of tight junction (TJ) proteins and inflammatory genes in colon and adipose tissues was examined. To determine if this protection against inflammation occurred in tissues, we also measured the inflammatory and anti-inflammatory cytokine secretion in the liver and white adipose tissues. Finally, the effects of different forms of A. muciniphila on the HSC activation and inflammatory markers were compared in vitro and in vivo.

\section{Materials and methods}

Bacterial culture, pasteurization and EV extraction. A. muciniphila MucT strain (ATCC BAA-835) (DSMZ institute, Germany) was cultured in a brain heart infusion (BHI) broth (QUELAB, Canada) supplemented with $0.5 \%$ mucin (SIGMA-ALDRICH, St Louis, MO, USA) at $37^{\circ} \mathrm{C}^{10}$. After the OD600 reached $\sim 1$ bacterial suspension was centrifuged at $11,000 \mathrm{~g}$ for $20 \mathrm{~min}$, the pellet was washed twice with sterile anaerobic PBS and used for further co-culture experiments and oral gavage in mice. For preparing pasteurized bacteria, a part of the bacterial suspension was also heated at $70{ }^{\circ} \mathrm{C}$ for $15 \mathrm{~min}$, as previously described ${ }^{14}$. For viability testing of the pasteurized $A$. muciniphila, the suspension was inoculated on a mucin-based medium and then incubated for at least 1 week at $37^{\circ} \mathrm{C}$ in the anaerobic atmosphere. The pasteurized bacteria were aliquot and stored at $-80^{\circ} \mathrm{C}$ until used. EVs were extracted with ultracentrifugation $\left(200,000 \mathrm{~g}\right.$ for $2 \mathrm{~h}$ at $\left.4{ }^{\circ} \mathrm{C}\right)$ of the bacterial culture supernatant aforementioned ${ }^{26}$.

LX-2 activation and treatment. The LX-2 cell line was kindly gifted from Professor Scott L. Friedman (Mount Sinai School of Medicine, New York, NY). LX-2 cells were maintained in complete Dulbecco's modified Eagle's medium supplemented with $2 \mathrm{mM}$ of L-glutamine, $100 \mathrm{U} / \mathrm{ml}$ of penicillin, $100 \mu \mathrm{g} / \mathrm{ml}$ of streptomycin, and 2\% heat-inactivated fetal bovine serum (GIBCO-INVITROGEN, Carlsbad, CA). For LX-2 activation, the lipopolysaccharides (LPS) from Escherichia coli 0111:B4 (SIGMA-ALDRICH) was used at concentration of $0.01 \mu \mathrm{g} / \mathrm{ml}$ for $6 \mathrm{~h}$ as previously described ${ }^{25}$. Afterwards, unstimulated LX-2 and LPS-activated cells were inoculated with live (Lam) and pasteurized (Pam) A. muciniphila at different multiplicities of infection (MOIs 1, 10, $100)$, and EVs of varying concentrations $(1,10,50 \mu \mathrm{g} / \mathrm{ml})$ for $24 \mathrm{~h}$ in the $\mathrm{CO}_{2}$ incubator at $37^{\circ} \mathrm{C}$. Each experiment was performed in duplicate, and repeated at least three times.

Total RNA extraction and qRT-PCR analysis. Total RNA was extracted from LX-2 cells using RNeasy Plus Mini Kit (QIAGEN, Germany) following the supplier's protocol. The quantity and quality of RNA was verified via agarose gel electrophoresis and NanoDrop spectrophotometer (ND-1000, THERMO SCIENTIFIC, USA). The purified RNA was converted to cDNA using the BIOFACT RT-Kit (BIOFACT, South Korea) according to the manufacturer's protocol. The qRT-PCR amplification was done on a ROTOR-GENE Q (QIAGEN, 


\begin{tabular}{|c|c|c|c|}
\hline Target gene & Primer designation & Oligonucleotide sequence $\left(5^{\prime}-3^{\prime}\right)$ & Product size (bp) \\
\hline $\mathrm{m}-r p l-19$ & $\begin{array}{l}\text { Rpl-F } \\
\text { Rpl-R }\end{array}$ & $\begin{array}{l}\text { TCAGCCACAACATTCTCA } \\
\text { GCACCTCCAACAGTAAGT }\end{array}$ & 138 \\
\hline $\mathrm{m}-t l r-5$ & $\begin{array}{l}\text { Tlr5-F } \\
\text { Tlr5-R }\end{array}$ & $\begin{array}{l}\text { AAGACTGCGATGAAGAGGAA } \\
\text { GGTGATGACGAGGAATAGAGT }\end{array}$ & 92 \\
\hline $\mathrm{m}-t l r-9$ & \begin{tabular}{|l|} 
Tlr9-F \\
Tlr9-R
\end{tabular} & $\begin{array}{l}\text { TCAGCCACAACATTCTCA } \\
\text { GCACCTCCAACAGTAAGT }\end{array}$ & 138 \\
\hline $\mathrm{m}-i l-1 \beta$ & $\begin{array}{l}\text { IL-1b-F } \\
\text { IL-1b-R }\end{array}$ & $\begin{array}{l}\text { AACAACTACTCAGAAACACAAG } \\
\text { GCAGAACTCAGGAATGGA }\end{array}$ & 130 \\
\hline m-il-6 & $\begin{array}{l}\text { IL6-F } \\
\text { IL6-R }\end{array}$ & $\begin{array}{l}\text { TCCATCCAGTTGCCTTCT } \\
\text { TAAGCCTCCGACTTGTGAA }\end{array}$ & 137 \\
\hline $\mathrm{m}-i l-10$ & $\begin{array}{l}\text { IL10-F } \\
\text { IL10-R }\end{array}$ & $\begin{array}{l}\text { GCACTACCAAAGCCACAAG } \\
\text { AGTAAGAGCAGGCAGCATAG }\end{array}$ & 85 \\
\hline $\mathrm{m}-i g f$ & $\begin{array}{l}\text { Igf-F } \\
\text { Igf-R }\end{array}$ & $\begin{array}{l}\text { TCGTGGGATGGGTGCTTT } \\
\text { TGAAGACAGTAGGGAAGAGACAAG }\end{array}$ & 154 \\
\hline $\mathrm{m}-$ ppar- $\alpha$ & $\begin{array}{l}\text { PPARa-F } \\
\text { PPARa-R }\end{array}$ & $\begin{array}{l}\text { CACTTGCTCACTACTGTCCTT } \\
\text { GATGCTGGTATCGGCTCAA }\end{array}$ & 110 \\
\hline $\mathrm{m}-p \operatorname{par}-\gamma$ & $\begin{array}{l}\text { PPARg-F } \\
\text { PPARg-R }\end{array}$ & $\begin{array}{l}\text { GGTGCTCCAGAAGATGACAGA } \\
\text { TCAGCGGGTGGGACTTTC }\end{array}$ & 154 \\
\hline $\mathrm{m}-$ ocldn & $\begin{array}{l}\text { Ocldn-F } \\
\text { Ocldn-R }\end{array}$ & $\begin{array}{l}\text { TTGAAAGTCCACCTCCTTACAGA } \\
\text { CCGGATAAAAAGAGTACGCTGG }\end{array}$ & 129 \\
\hline $\mathrm{m}-\mathrm{cldn}-1$ & $\begin{array}{l}\text { Cldn1-F } \\
\text { Cldn1-R }\end{array}$ & $\begin{array}{l}\text { TCTGCCACTTCTCACTTCCA } \\
\text { GCCTATACCCTTGCTCTCTGT }\end{array}$ & 95 \\
\hline $\mathrm{m}-\mathrm{cldn}-2$ & $\begin{array}{l}\text { Cldn2-F } \\
\text { Cldn2-R }\end{array}$ & $\begin{array}{l}\text { TGGAAGATGGTGATGGGATT } \\
\text { CCCTTGGAAAAGCCAACCG }\end{array}$ & 128 \\
\hline$g a p d h$ & $\begin{array}{l}\text { GAPDH-F } \\
\text { GAPDH-R }\end{array}$ & $\begin{array}{l}\text { TCAACGGATTTGGTCGTATTG } \\
\text { TGGAAGATGGTGATGGGATT }\end{array}$ & 211 \\
\hline ppar-a & $\begin{array}{l}\text { PPARa-F } \\
\text { PPARa-R }\end{array}$ & $\begin{array}{l}\text { GAGCGTTGTCTGGAGGTT } \\
\text { GAAGTGGTGGCTAAGTTGTTG }\end{array}$ & 115 \\
\hline ppar- $\gamma$ & $\begin{array}{l}\text { PPARg-F } \\
\text { PPARg-R }\end{array}$ & $\begin{array}{l}\text { TACGAAGACATTCCATTCACAAGA } \\
\text { CTCCACAGACACGACATTCA }\end{array}$ & 199 \\
\hline$t l r-5$ & \begin{tabular}{|l|} 
Tlr5-F \\
Tlr5-R \\
\end{tabular} & $\begin{array}{l}\text { TCCCTGAACTCACGAGTCTTT } \\
\text { GGTTGTCAAGTCCGTAAAATGC }\end{array}$ & 109 \\
\hline $\operatorname{tr}-9$ & $\begin{array}{l}\text { Tlr9-F } \\
\text { Tlr9-R }\end{array}$ & $\begin{array}{l}\text { CTGCCACATGACCATCGAG } \\
\text { GGACAGGGATATGAGGGATTTGG }\end{array}$ & 121 \\
\hline
\end{tabular}

Table 1. Oligonucleotide primers used in real-time PCR in cell line and mice tissues.

Germany) real-time PCR system by using BIOFACT 2X Real-Time PCR Master Mix (BIOFACT, South Korea). The GAPDH served as the reference gene. The oligonucleotide sequences used in this work are presented in Table 1.

Animal experiments. All animal studies were carried out in compliance with the ARRIVE guidelines. Twenty-five male wild-type C57BL/6 mice were obtained at the age of 8 weeks from Pasteur Institute of Iran (Tehran Iran). Acclimatization was performed in $12 \mathrm{~h}$ light, $22-23^{\circ} \mathrm{C}$, and $40 \%$ humidity condition with a free access to standard diet (SD) (Standard Rodent Diet A03; SAFE, Augy, France) and autoclaved water. Then, mice were individually housed in autoclaved cages and sterile hardwood chip bedding during the experiment. This study followed the institutional guidelines regarding the care and use of laboratory animals. The study protocol was approved by the Animal Experiment Committee Pasteur Institute of Iran (IR.PII.REC.1399.029).

For liver injury induction, $\mathrm{CCl}_{4}$ accompanied by a high-fat diet $(260 \mathrm{HF} 60 \%$ energy from butter, safe diet, France) was used. The HFD animals were intraperitoneally injected with $2 \mathrm{ml} / \mathrm{kg}$ body weight of $10 \% \mathrm{CCl} 4$ solution in olive oil (SIGMA-ALDRICH, St. Louis, MO, USA) twice a week for 4 weeks ${ }^{27}$. Mice were randomly grouped $(n=5)$, healthy control animals received the SD without intervention, vehicle (HFD/CCL4+200 $\mu$ l sterile PBS), Lam (HFD/CCL $4+10^{9} \mathrm{CFU} / 200 \mu \mathrm{l}$ live A. muciniphila), Pam (HFD/CCL $4+10^{9} \mathrm{CFU} / 200 \mu$ l pasteurized $A$. muciniphila) and EV (HFD/CCL $4+50 \mu \mathrm{g}$ protein/200 $\mu \mathrm{l})$ were given by daily oral gavage for 4 weeks (Fig. $1 \mathrm{~A}$ ). At the end of the treatments, the mice were sacrificed by cervical dislocation and liver, colon and white adipose tissues were snap frozen with liquid nitrogen, and stored at $-80^{\circ} \mathrm{C}$. The liver and colon tissues were further used for histopathological analysis and blood samples were taken without prior food fasting, serum was collected and stored at $-80^{\circ} \mathrm{C}$ until further analysis.

Histopathological evaluation and histometric analysis. Liver and colon specimens were immediately fixed in $10 \%$ neutral buffered formalin. Paraffin-embedded liver and colon tissues were stained with hematoxylin and eosin (H\&E) method ${ }^{28}$. The thickness of the epithelium and mucosal layer were then evaluated by an expert pathologist, blind to the study groups. Histological pictures of colon and liver were taken using a light microscope (OLYMPUS SX-21) equipped with a digital camera (TRUECHROME II).

For histometrical study, Dino Lite digital lens and Dino Capture 2 Software and light microscope were used. Furthermore, histometrical structures of colon were analyzed, including thickness of mucosal layer and the 

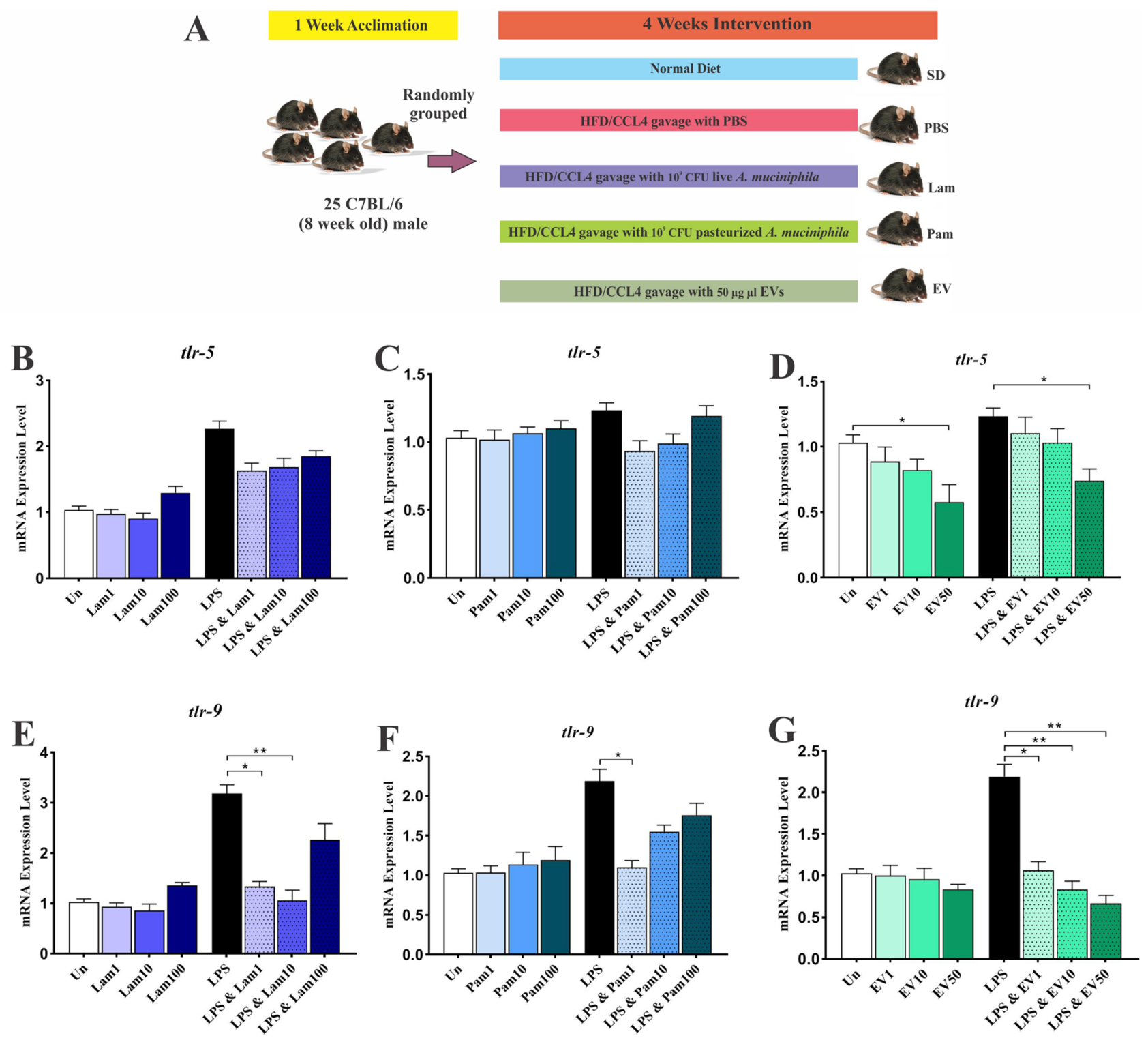

Figure 1. (A) Study design of the animal experiment. Anti-inflammatory effects of all A. muciniphila supplementations in LX-2 cell line. The mRNA Level of $t r-5$ in quiescence and LPS-activated LX-2 cells treated with (B) Lam (C) Pam and (D) EVs; and tlr-9 in quiescence and LPS-activated LX-2 cells treated with (E) Lam (F) Pam and (G) EVs. Un: untreated cells, Lam: live A. muciniphila, Pam: pasteurized A. muciniphila, EV: extra cellular vesicles of $A$. muciniphila. Data are expressed as mean $\pm \operatorname{SD}(n=5) .{ }^{*} p<0.05,{ }^{* *} p<0.01$ and ${ }^{* * *} p<0.001$ by post hoc Turkey's one-way ANOVA statistical analysis.

epithelium. A microscopic histologic injury score was assigned to each mouse. Scoring was based on infiltration of inflammatory cells. Each category was scored with a score of 0 indicating no infiltration of inflammatory cells and a score of 3 indicating infiltration of inflammatory cells $\mathrm{s}^{29,30}$. Number of bi-nucleated hepatocytes per $\mathrm{mm} 2$ was calculated in 20 randomly selected fields of each section using Dino Lite digital lens and Dino Capture 2 Software and light microscope ${ }^{31}$.

Measurement of serum aminotransferases and liver cytokine. Serum level of alanine transaminase (ALT) and aspartate transaminase were assessed to show the degree of liver injury using commercial assay kits according to the instructions of manufacturers. The level of IL-10, TNF- $\alpha$ and IL- 6 in liver and adipose tissues were determined by using ZELLBIO GmbH ELISA kit (Germany) according to the manufacturer's instructions, and analyzed with Bio-Plex Manager 6.1 software (BIO-RAD, USA). This cytokine assays were performed in duplicate.

Tissue RNA extraction and qRT-PCR analysis. Frozen liver, colon and white adipose tissues were homogenized in $1 \mathrm{ml}$ of TRIZOL (33 BS410, Bio Basic, Canada) using a Precellys 24homogenizer and the total RNA was extracted according to the manufacturer's instructions. The genomic DNA was removed using DNase 
I (QIAGEN), cDNA was synthesized and qRT-PCR amplification was carried out as mentioned earlier. The relative expression of target genes was assessed using the comparative cycle threshold (Ct) method. The rpl-19 served as the normalizer genes.

Statistical analysis. GRAPHPAD PRISM 8.0 (GRAPHPAD Software Inc, CA, United States) was exploited for calculating changes in gene expression and cytokine production. Differences between groups were calculated using one-way analysis of variance (ANOVA) followed by Tukey's post hoc test (for multiple comparisons between more than two groups). Results are shown as mean \pm SD (standard deviation) of the mean of at least three experiments. A $p$ value less than 0.05 were considered statistically significant.

Approval for animal experiments. The study was approved by the Animal Experiment Committee of Pasteur Institute of Iran (IR.PII.REC.1399.029), and the Institutional Ethical Review Committee of Research Institute for Gastroenterology and Liver Diseases at Shahid Beheshti University of Medical Sciences (Project No. IR.SBMU.RIGLD.REC.1395.211).

\section{Results}

A. muciniphila attenuates mRNA level of $t / r-5$ and $t / r-9$ in LPS-activated LX-2 cells. To evaluate the effects of $A$. muciniphila and its derivatives on mRNA levels of $t l r-5$ and $t l r-9$ genes, different MOIs $(1,10$, and 100) and concentration of EVs $(1,10$, and $50 \mu \mathrm{g})$ were used in quiescent and LPS-activated LX-2 cells. As shown in Fig. 1B-G, in quiescent LX-2 cells, none of the live and pasteurized treatments altered the expression of tlr- 5 and $t l r-9$ genes, while EVs in a concentration of $50 \mu \mathrm{g}$ had an inhibitory effect on the expression of $t l r-5$ in comparison with untreated control cells ( $p$ value 0.03 ). In LPS-stimulated LX-2 cells also, just EV in concentrations of $50 \mu \mathrm{g}$ could significantly reduce $t r-5$ gene mRNA level ( $p$ value 0.012 ). In LPS-activated LX-2 cells, all concentrations of EV were able to down-regulate the $t l r-9$ gene significantly ( $p$ value $0.04,0.003$ and 0.001 for 1 , 10 , and $50 \mu$ g respectively), while Lam at MOI 1 ( $p$ value 0.05$)$ and 10 ( $p$ value 0.02$)$ and Pam at MOI1 ( $p$ value $0.05)$ could reduce the expression of $t r-9$ gene. Therefore, although all treatments had an inhibitory effect on the mRNA level of the $t l r-5$ and $t l r-9$ genes in the activated LX- 2 cells, the EVs effects were more pronounced and almost no significant effect was observed on the quiescent cells.

A. muciniphila significantly up-regulates PPARs gene expression in LX-2 cells. The effect of live and pasteurized A. muciniphila and its EVs on gene expression of anti-inflammatory factor ppar- $\alpha$ and ppar- $\gamma$ was also investigated in quiescent and LPS-stimulated LX-2 cells. As shown in Fig. 2 in quiescent cells, treatment with different live and pasteurized bacterial MOIs and EV concentrations did not significantly alter the expression of the ppar- $\alpha$ and ppar- $\gamma$ gene, whereas EV in concentrations of $50 \mu \mathrm{g}$ was able to significantly increase the mRNA level of ppar- $\alpha$ and ppar- $\gamma$ ( $p$ value 0.01 and 0.03 respectively).

In LPS-activated cells, a significant mRNA level of ppar- $\alpha$ gene was induced compared to untreated control by different treatments. Among them, Lam and EV at all three MOI and concentrations and Pam at MOI10 and 100 showed a significant effect on induction of ppar- $\alpha$ gene (Fig. 2A-C). However, in LPS-activated LX-2 cells, a significant mRNA level of ppar- $\gamma$ was induced by different treatments compared to untreated control cells, among which bacterial MOI10 and EV 50 showed a significant effect on induction of $p$ par- $\gamma$ gene ( $p$ value $<0.0001$ and $<0.0001$ respectively) (Fig. 2D-F). Thus, almost all treatments were able to induce the expression of ppar- $\alpha$ and ppar- $\gamma$ genes, although the best effect was observed in EV treatments in a dose-dependent manner.

A. muciniphila and its derivatives could maintain intestinal homeostasis by modulating inflammation. As shown in Fig. 3A, the PBS group revealed the infiltration of inflammatory cells (Grade 2 ) in the mucous membrane of the colon. The thickness of epithelium showed a significant decrease in PBS group $(11.92 \pm 1.64 \mu \mathrm{m})$, compared to that in SD group $(16.7 \pm 0.37 \mu \mathrm{m})(p$ value $<0.05)$ (Fig. 3B). The thickness of mucosal layer showed a significant decrease in PBS group $(92.58 \pm 7.8 \mu \mathrm{m})$, compared to that in SD group $(119.95 \pm 1.97 \mu \mathrm{m})(p$ value $<0.05)$. The maximum thickness of mucosal layer $(161.45 \pm 14.5 \mu \mathrm{m})$ and epithelium $(21.05 \pm 1.25 \mu \mathrm{m})$ was shown in Pam group (Fig. 3C). Furthermore, the thickness of mucosal layer and epithelium showed a significant increase in Lam and EV, compared to that in PBS group ( $p$ value $<0.05)$. As demonstrated in Fig. 3D, Crypt depth showed a significant increase in EV $(119.9 \pm 3.1 \mu \mathrm{m})$, Pam $(148.9 \pm 1.8 \mu \mathrm{m})$ and Lam $(135.1 \pm 3.2 \mu \mathrm{m})$ groups, compared to that in PBS group ( $p$ value $<0.000)$. Furthermore, crypt depth showed a significant increase in Pam and Lam groups, compared to that in SD group $(116.8 \pm 1.6 \mu \mathrm{m})(p$ value $<0.05)$. Moreover, the crypt depth in the colon showed a significant decrease in PBS group $(86.9 \pm 1.6 \mu \mathrm{m})$, compared to that in all groups $(p$ value $<0.000)$.

By using a histopathological score system, as described previously ${ }^{30}$ histologic injury score of the mice colon tissue was categorized according to the following characterizes: epithelial tissue damage, infiltration of inflammatory cells, hemorrhagic congestion and edema of the mucosa in the three categories range from 0 (no injury) to 3 (severe injury). The overall score was the sum of each component score (Fig. 3E). The total score of each group (Mean \pm SE) was as follows: SD group, $0.42 \pm 0.22$; PBS group, $4.25 \pm 0.14$; Lam group, $2.42 \pm 0.22$; Pam group, $1.25 \pm 0.14$ and EV group $0.58 \pm 0.08$. Statistical analysis of the histopathological score showed the following: $\mathrm{PBS}$ vs SD with $p$ value $<0.000$; Lam vs $\mathrm{PBS}$ with $p$ value $<0.000$; Pam vs $\mathrm{PBS}$ with $p$ value $<0.000$; and EV vs PBS with $p$ value $<0.000$.

These observations were accompanied by a significant increase in mRNA level of tight junction proteins ocldn and $c l d n-1$ and a decrease in cldn-2 in the colon of mice treated with live and pasteurized A. muciniphila and its EVs (Fig. 3 F-H). Although there was a significant difference in the thickness of mucosal layer between treatments, their ability to induce tight junction protein genes was the same. 

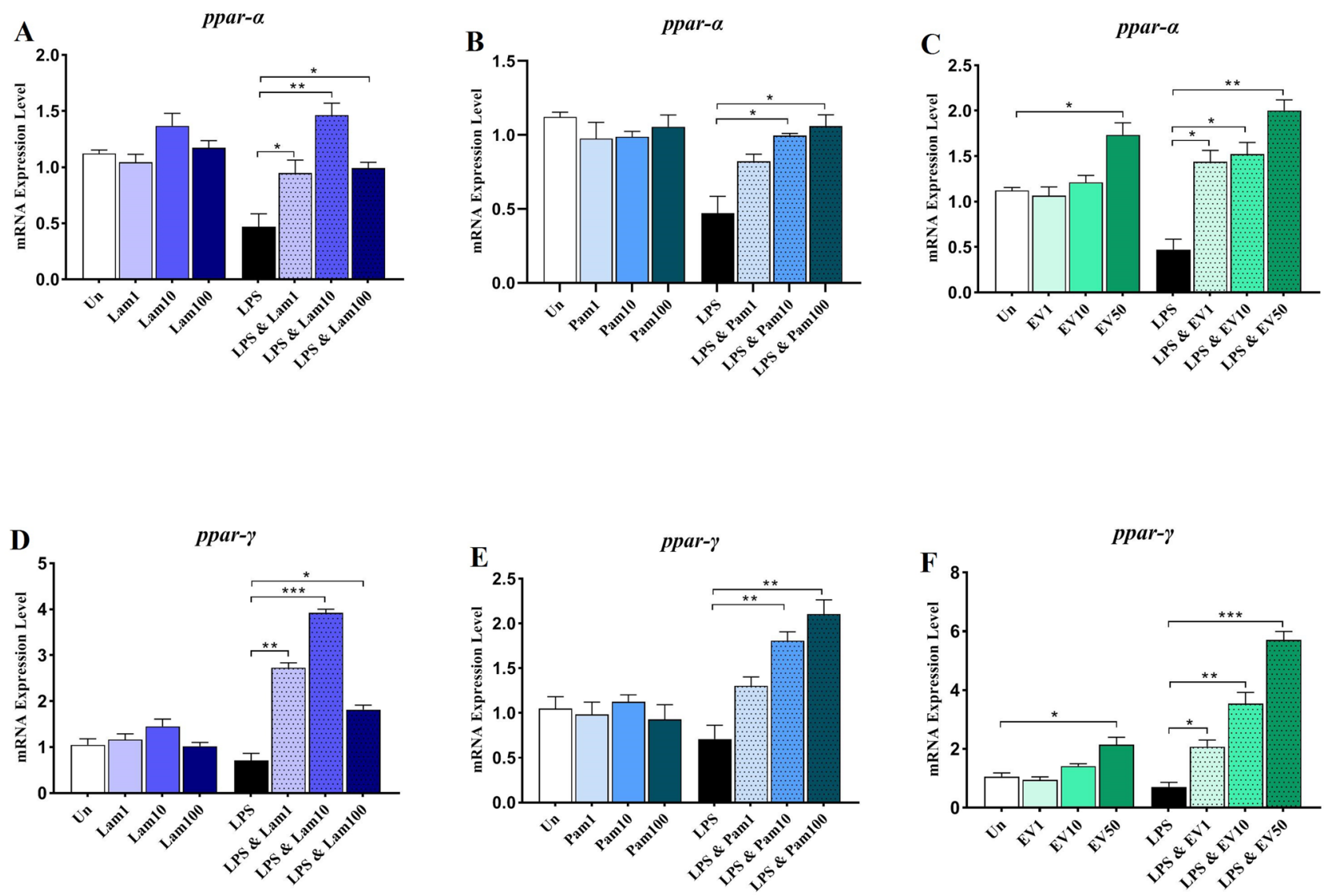

Figure 2. Anti-inflammatory effects of all A. muciniphila supplementations in LX-2 cell line. The mRNA Level of ppar- $\alpha$ in quiescence and LPS-activated LX-2 cells treated with (A) Lam (B) Pam and (C) EVs; and ppar- $\gamma$ in quiescence and LPS-activated LX-2 cells treated with (D) Lam (E) Pam and (F) EVs. Un: untreated cells, Lam: live A. muciniphila, Pam: pasteurized A. muciniphila, EV: extra cellular vesicles of $A$. muciniphila. Data are expressed as mean $\pm \mathrm{SD}(n=5) .{ }^{\star} p<0.05,{ }^{* *} p<0.01$ and ${ }^{* * *} p<0.001$ by post hoc Turkey's one-way ANOVA statistical analysis.

Live A. muciniphila and its EVs had anti-inflammatory effects on liver tissue of HFD/ CCL4-treated mice. Liver histopathological analysis confirmed that acute liver injury was established in PBS group comparing to SD group (Fig. 4A). In H\&E staining, no histopathological changes were observed in SD group, while infiltration of inflammatory cells in the form of spotty necrosis and necrosis of hepatocytes along with the lipid micro and macro vesicles in the cytoplasm of hepatocytes was observed in PBS group. As shown in Fig. 4B, the number of bi-nucleated hepatocytes showed a significant decrease in PBS group, compared to that in SD group $(p$ value $<0.05)$. Furthermore, number of bi-nucleated hepatocytes showed a significant increase in EV, Lam and Pam groups, compared to that in SD as well as PBS groups ( $p$ value $<0.05$ and $<0.05$, respectively). The presence of bi-nucleated hepatocyte cells indicated hepatocyte regeneration without any other histopathological changes. In Pam group, although infiltration of inflammatory cells, spotty necrosis, necrosis of hepatocytes, and lipid micro and macro vesicles was observed in the cytoplasm of hepatocytes, the presence of bi-nucleated hepatocyte cells indicating hepatocyte regeneration was also observed. In addition, by using a histopathological score system, as described previously ${ }^{32,33}$ histologic injury score of the liver tissue was categorized according to the following characterizes: necrosis, infiltration of inflammatory cells, steatosis in the four categories range from 0 (no) to 4 (severe). The overall score was the sum of each component score (Fig. 4C). The total score of each group (Mean \pm SE) was as follows: SD group, $0.5 \pm 0.14$; PBS group, $9.5 \pm 0.28$; Lam group, $1.83 \pm 0.16$; Pam group, $6.83 \pm 0.44$ and EV group $0.75 \pm 0.13$. Statistical analysis of the histopathological score showed the following: PBS vs SD with $p$ value $<0.000$; Lam vs PBS with $p$ value $<0.000$; Pam vs PBS with $p$ value $<0.000$; and EV vs PBS with $p$ value $<0.000$. In addition, there were no significant differences observed between $\mathrm{EV}$ vs SD.

To investigate the anti-inflammatory effects of live and pasteurized A. muciniphila and its EVs, the level of TNF- $\alpha$, IL-6, and IL-10 in mice liver tissue was also assessed. As shown in Fig. 4D-F, asignificant increase in the level of IL-6 ( $p$ value 0.0006 ), TNF- $\alpha$ ( $p$ value 0.001 ) and decrease in IL-10 ( $p$ value 0.0002 ) was observed in PBS group compared to SD group indicating inflammation was induced in the liver tissue of $\mathrm{HFD} / \mathrm{CCL}_{4}$ treated mice. Although oral gavage with live A. muciniphila could decrease TNF- $\alpha$, this effect was not statistically significant ( $p$ value 0.1 ), while administration of EVs could remarkably decrease the level of TNF- $\alpha$ ( $p$ value 0.01$)$. Moreover, oral gavage with live A. muciniphila and its EVs could significantly decrease the level of IL-6 ( $p$ value 


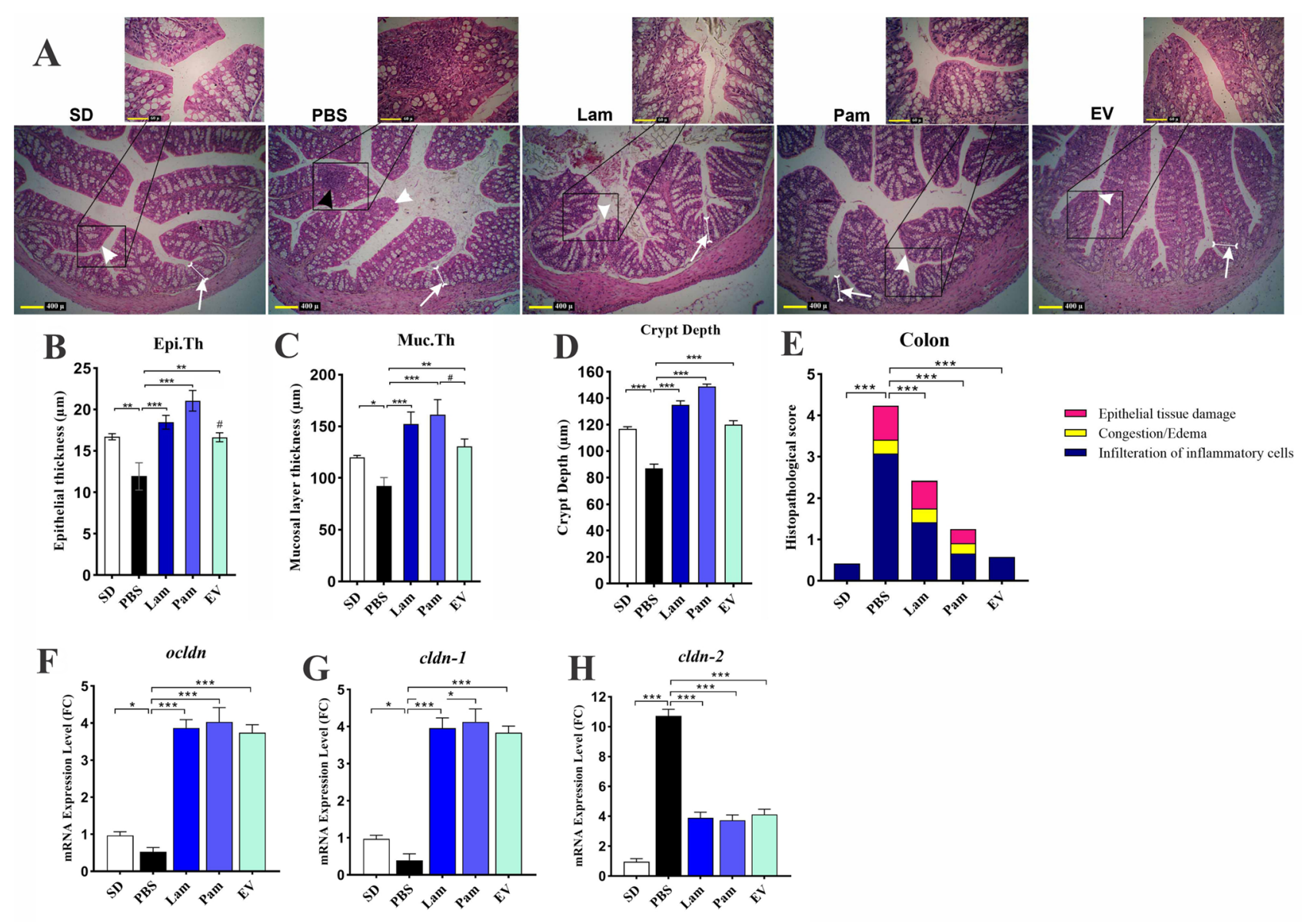

Figure 3. (A) Histological structure of colon in different study groups using H\&E staining. The infiltration of inflammatory cells (black arrow) was observed in the mucous membrane of the PBS group. The decrease in thickness of mucosal layer (white arrows) and thickness of the epithelium (white arrowheads) of the colon were seen in histological sections of PBS group. An increase in thickness of the epithelium (white arrowheads) and thickness of mucosal layer (white arrows) was seen in Pam, Lam, and EV groups, compared to that in PBS group. histometric analysis shows the difference of (B) epithelium thickness (Epi.Th) and (C) the mucosal layer thickness (Muc.Th) (D) crypt depth and (E) histopathological score among the study groups. Assessment of a live and pasteurized A. muciniphila and its EVs effects on mRNA expression of tight junction proteins gene in the colon tissue. (F) ocldn, (G) cldn-1 and (H) cldn-2. SD: standard diet, PBS: HFD/CCL4+ PBS, Lam: HFD/ CCL4+ A. muciniphila (109 CFU), Pam: HFD/CCL4+ pasteurized A. muciniphila (109 CFU) and EV: HFD/ CCL4 $+50 \mu \mathrm{g}$ EVs. Data are expressed as mean $\pm \mathrm{SD}(n=5) .{ }^{*} p<0.05,{ }^{* *} p<0.01,{ }^{* *} p<0.001$ in comparison with PBS group and \#\# $p<0.01$ in comparison with Pam by post hoc Turkey's one-way ANOVA statistical analysis.

0.001), while pasteurized form could not significantly change in IL-6 level compared to PBS group. The effect of pasteurized A. muciniphila also was not significant on TNF-a cytokines, while it significantly increased the tissue level of IL-10( $p$ value 0.03 ). Although oral gavage with all treatments could decrease the level of IL-6 and TNF- $\alpha$ and increase IL-10, these anti-inflammatory effects were remarkable in Lam and EV groups.

Moreover, assessment of serum aminotransferases level showed a significant increase in ALT and AST level of the PBS in comparison with the SD group ( $p$ value $<0.0001$ ), indicating liver injury was established in PBS group. As shown in Table 2, oral gavage with Lam, Pam, and EVs was able to decrease the level of ALT, AST in study groups.

\section{A. muciniphila demonstrates inhibitory effect on inflammatory genes in liver tissue of HFD/} CCL4-treated mice. In the present study, the anti-inflammatory effect of live and pasteurized A. muciniphila and its EVs was investigated by inhibiting the expression of TLRs and inflammatory cytokines such as $i l-1 \beta$, il-6, and anti-inflammatory il-10 in the liver tissue of the $\mathrm{HFD} / \mathrm{CCL}_{4}$ murine model.

As shown in Fig. 5 A-E, HFD/CCL 4 remarkably elevated $t l r-5$ and $t r-9$ mRNA levels which in turn led to an increase in inflammatory cytokines and a decrease in $i l-10$ in the PBS group ( $p$ value $<0.0001$ ). Administration of Lam, Pam, and EVs were able to significantly decrease the expression of $t l r-5$ and $t l r-9$ genes compared to the PBS group ( $p$ value $<0.0001,<0.0001$ and $<0.0001$, respectively). This bacterium and its derivatives also regulated the inflammatory and anti-inflammatory cytokines by reducing the expression of $i l-1 \beta$ and $I L-6$ and increasing 
A

SD

PBS

Lam

Pam

EV
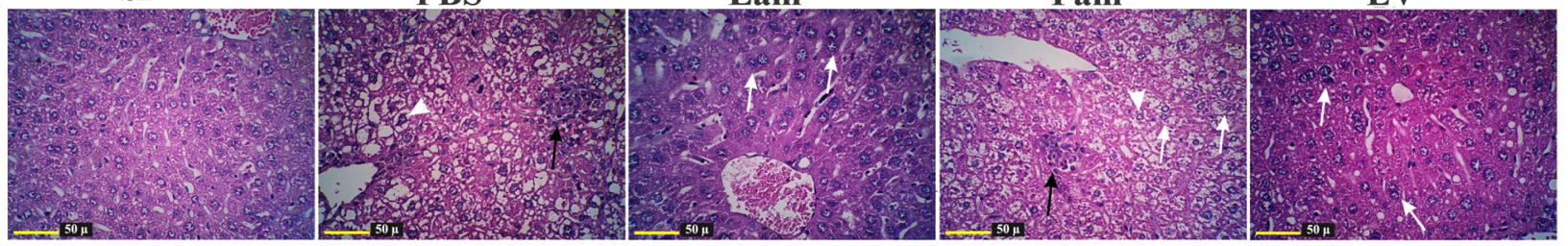

B Bi-nucleated hepatocytes

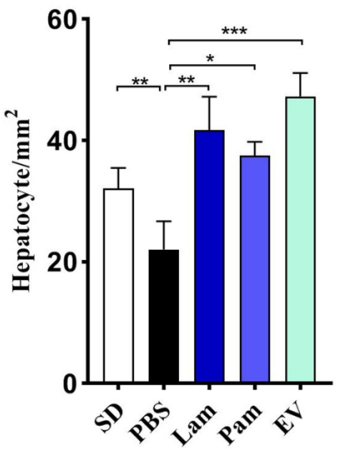

C

Liver
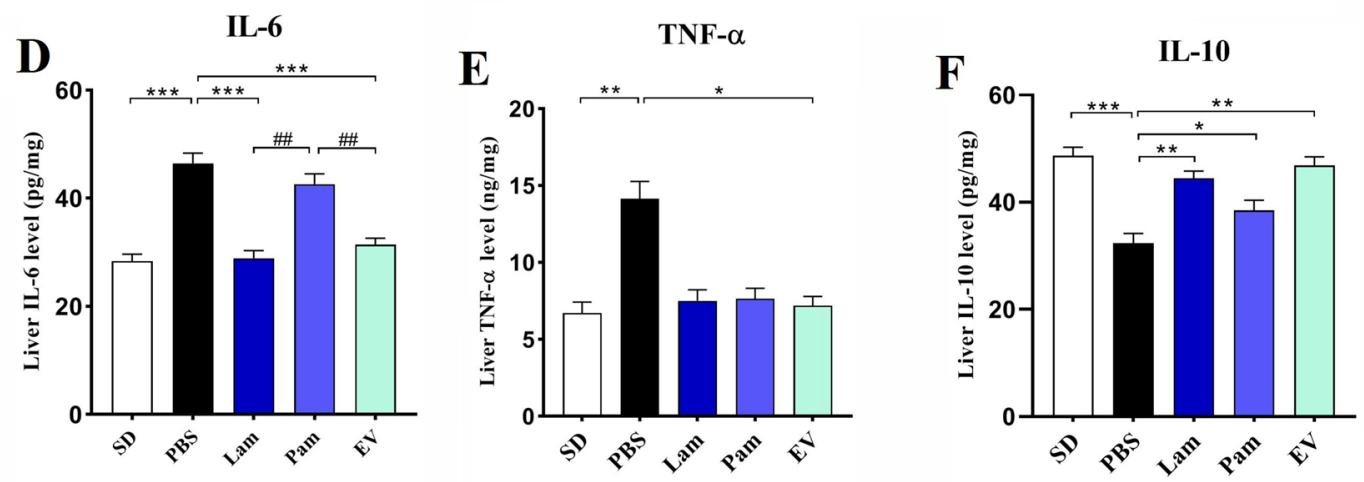

Figure 4. (A) The effect of a live and pasteurized A. muciniphila administration on the histopathological structure of the liver in different study groups (H\&E). In the PBS group, infiltration of inflammatory cells and spotty necrosis of hepatocytes (Black arrows), lipid micro and macro vesicles (White arrowheads) were presented in the cytoplasm of hepatocytes. Lam and EV groups: without histopathological changes and the presence of bi-nucleated cells (white arrows). Pam group: infiltration of inflammatory cells and spotty necrosis of hepatocytes (Black arrows), lipid micro and macro vesicles (White arrowheads) and the presence of bi-nucleated cells (white arrows). The number of bi-nucleated hepatocytes in histological structure of liver in different study groups (mean \pm SD). (B) Counting of bi-nucleated hepatocytes per $\mathrm{mm} 2(\mathbf{C})$ histopathological score. The liver tissue cytokines level (D) IL-6, (E) TNF- $\alpha$ and (F) IL-10. SD: standard diet, PBS: HFD/ CCL4 + PBS, Lam: HFD/CCL4 + A. muciniphila (109 CFU), Pam: HFD/CCL4 + pasteurized A. muciniphila (109 CFU) and EV: HFD/CCL4 + $50 \mu \mathrm{g}$ EVs. Data are expressed as mean $\pm \mathrm{SD}(n=5){ }^{\star} p<0.05,{ }^{* *} p<0.01,{ }^{* * *}$ $p<0.001$ in comparison with PBS group and \#\# $p<0.01$ in comparison with Pam by post hoc Turkey's one-way ANOVA statistical analysis.

\begin{tabular}{|c|c|c|c|c|c|c|c|c|c|}
\hline \multirow[b]{2}{*}{ Variables } & \multicolumn{5}{|c|}{ Study groups $($ mean $\pm \mathrm{SD})$} & \multicolumn{4}{|l|}{$P$ value } \\
\hline & SD & PBS & Lam & Pam & EV & SD vs. PBS & $\begin{array}{l}\text { Lam vs. } \\
\text { PBS }\end{array}$ & $\begin{array}{l}\text { Pam vs. } \\
\text { PBS }\end{array}$ & EV vs. PBS \\
\hline $\operatorname{ALT}(\mathrm{U} / \mathrm{dl})$ & $71.00 \pm 6.43$ & $162.00 \pm 6.24$ & $85.18 \pm 9.08$ & $121.3 \pm 5.84$ & $84.18 \pm 6.67$ & $<0.0001$ & $<0.0001$ & 0.002 & $<0.0001$ \\
\hline AST (U/dl) & $86.74 \pm 3.88$ & $181.9 \pm 5.49$ & $83.82 \pm 6.12$ & $92.26 \pm 5.23$ & $85.16 \pm 4.86$ & $<0.0001$ & $<0.0001$ & $<0.0001$ & $<0.0001$ \\
\hline
\end{tabular}

Table 2. The effect of a live and pasteurized A. muciniphila and its EVs administration on serum level of aminotransferases after 4 weeks ( $n=5$ for each group). SD; standard-diet, Lam; live A. muciniphila, Pam; pasteurized A. muciniphila, EV; extracellular vesicle, ALT; alanine aminotransferase, AST; aspartate aminotransferase, Bold $p$ value are indicated statically significant. 

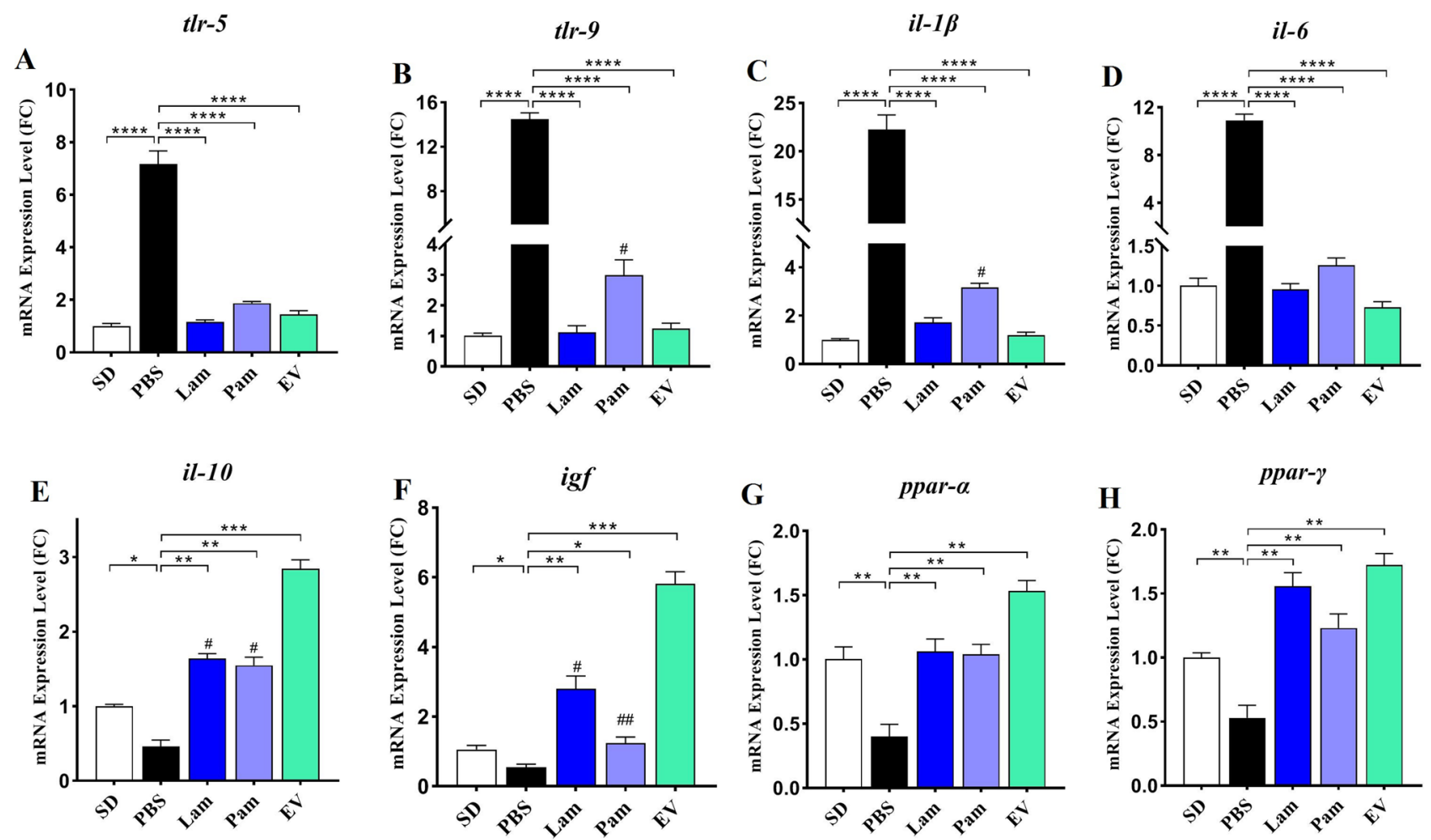

Figure 5. Hepatic mRNA expression of inflammatory-related genes, (A) tlr-5; (B) tlr-9; (C) $i l-1 \beta$; (D) il-6; and anti-inflammatory-related genes (E) il-10; (F) igf; (G) ppar- $\alpha$ and (H) ppar- $\gamma$. SD: standard diet, PBS: HFD/ CCL4+ PBS, Lam: HFD/CCL4 + A. muciniphila ( $\left.10^{9} \mathrm{CFU}\right)$, Pam: HFD/CCL4 + pasteurized A. muciniphila $\left(10^{9} \mathrm{CFU}\right)$ and EV: HFD/CCL4 $+50 \mu \mathrm{g}$ EVs. Data are expressed as mean $\pm \mathrm{SD}(n=5) .{ }^{*} p<0.05,{ }^{* *} p<0.01$ and ${ }^{* * *} p<0.001$ in comparison with PBS group, $\# p<0.05$ and $\# \# p<0.01$ in comparison with EV by post hoc Turkey's one-way ANOVA statistical analysis.

the $i l-10$ consequently induced immune homeostasis in liver tissue. Interestingly, EV showed the highest effect on $i l-10$ induction and $i l-1 \beta$ inhibition between the groups.

A. muciniphila shows anti-inflammatory effects on liver tissue of HFD/CCL4-treated mice through up-regulating of PPARs gene expression. The effect of live and pasteurized A. muciniphila and its EVs was investigated on gene expression of anti-inflammatory factors ppar- $\alpha$, ppar- $\gamma$ and igf in liver tissue. As shown in Fig. $5 \mathrm{~F}-\mathrm{H}$, ppar- $\alpha$, ppar- $\gamma$, and igf expression were significantly reduced in the PBS group which hepatic injury was induced by $\mathrm{HFD} / \mathrm{CCL}_{4}$. Oral gavage with all treatments was able to considerably induced the mRNA level of these anti-inflammatory factors ppar- $\alpha$, ppar- $\gamma$, and igf in liver tissue of study groups in comparison with PBS group, whereas the effect of EVs was more noticeable than live and pasteurized $A$. muciniphila especially in case of igf and ppar- $\alpha$ ( $p$ value $<0.0001$ and $<0.0001)$.

EVs had a remarkable anti-inflammatory effect on white adipose tissue of $\mathrm{HFD} / \mathrm{CCL}_{4}$-treated mice. To investigate the anti-inflammatory effects of live and pasteurized A. muciniphila and its EVs, the level of TNF- $\alpha$, IL-6, and IL-10 in mice adipose tissue was assessed. As shown in Fig. 6 A-C, a significant increase in level of IL-6 ( $p$ value 0.01 ) and TNF- $\alpha$ ( $p$ value 0.02 ) was observed in PBS group compared to SD group indicating inflammation was induced in adipose tissue. Although oral gavage with live and pasteurized A. muciniphila could decrease IL-6, this effect was not statistically significant, while administration of EVs could remarkably decrease the level of IL-6 ( $p$ value 0.0003). Oral gavage with live A. muciniphila and its EVs were also decreased TNF- $\alpha$ ( $p$ value 0.008 and 0.0004 respectively) and increased IL-10 ( $p$ value 0.009 and 0.0001 respectively). The effect of pasteurized A. muciniphila on TNF- $\alpha$ and IL- 6 was not statically significant although IL-10 was significantly changed ( $p$ value 0.04 ). Although oral gavage with all treatments could decrease the level of IL-6 and TNF- $\alpha$ and increase IL-10, these anti-inflammatory effects were remarkable in Lam and EV groups.

The anti-inflammatory effects of A. muciniphila and its EVs were accompanied by an increase in the mRNA level of ppar- $\alpha$ and ppar- $\gamma$ along with decrease in $i l-1 \beta$ in mice white adipose tissue. Although live and pasteurized A. muciniphila was able to enhance the expression of $p p a r-\alpha$, the effect of EVs was more noticeable $(p$ value $<0.0001)$. All treatments had significant increasing effect on $p p a r-\gamma$ and decreasing $i l-1 \beta$ in adipose tissue, while administration of $\mathrm{HFD} / \mathrm{CCL}_{4}$ remarkably down-regulated ppar- $\alpha$ and $p$ par- $\gamma$ and up-regulated $i l-1 \beta$ in PBS group (Fig. 6 D-F). 

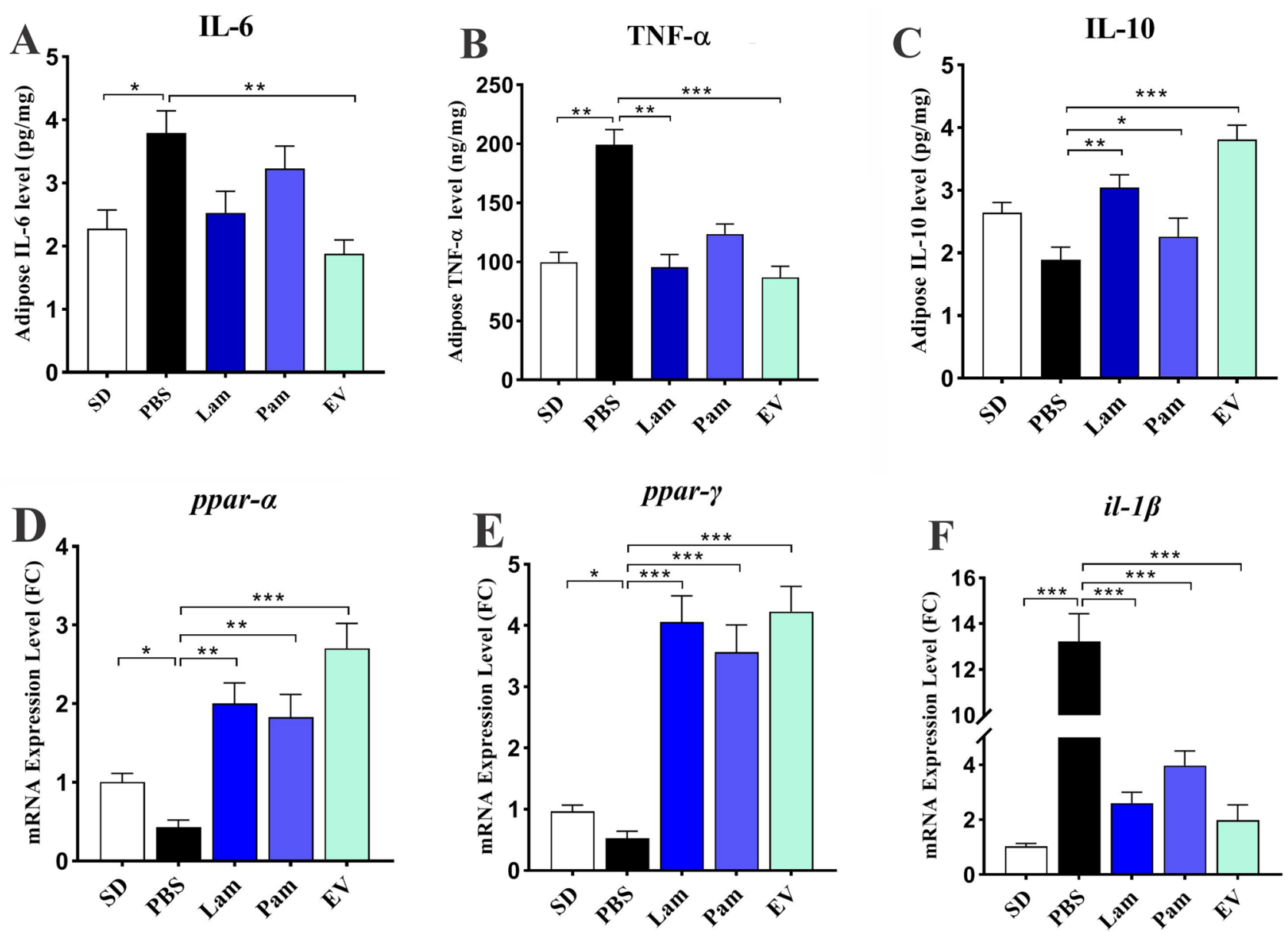

Figure 6. The adipose tissue cytokines level (A) IL-6; (B) TNF- $\alpha$ and (C) IL-10. Adipose tissue mRNA level of (G) ppar-a, (H) ppar- $\gamma$ and (C) il-1 $\beta$. SD: standard diet, PBS: HFD/CCL4+ PBS, Lam: HFD/CCL4 + A. muciniphila $\left(10^{9} \mathrm{CFU}\right)$, Pam: HFD/CCL4 + pasteurized $A$. muciniphila $\left(10^{9} \mathrm{CFU}\right)$ and EV: HFD/CCL4 $+50 \mu \mathrm{g}$ EVs. Data are expressed as mean $\pm \operatorname{SD}(n=5) .{ }^{\star} p<0.05,{ }^{* *} p<0.01$ and ${ }^{\star * *} p<0.001$ by post hoc Turkey's oneway ANOVA statistical analysis.

\section{Discussion}

Liver inflammation is considered the main cause of liver disease and hepatic tissue damage, leading to the progression of liver fibrosis and eventually $\mathrm{HCC}^{34}$. The main pathological mechanism underlying systemic inflammation is disruption of the intestinal epithelial integrity due to a decrease in TJ proteins. Liver is the first organ to be affected by this disturbance, as seen in the pathophysiology of different liver diseases ${ }^{35,36}$. The results of this study showed that four weeks of oral gavage with live and pasteurized A. muciniphila and its EVs could successfully maintain homeostasis in the colon tissue and increase mucosal thickness and crypt depth. This improvement in the histopathology of colon tissue was accompanied by an increase in RNA level of TJ proteins, resulting in the improvement of liver histopathology in an $\mathrm{HFD} / \mathrm{CCL}_{4}$-induced mouse model.

In a previous study conducted by our team of researchers, it was found that $A$. muciniphila and its EVs reduced the adipose size, inhibited inflammation, and increased mucosal thickness and crypt depth in the colon tissue of HFD-induced obese mice ${ }^{37}$. Grander et al. also found that three doses of live A. muciniphila could reduce liver damage and restore the intestinal barrier integrity in mice with alcohol-induced liver injury ${ }^{38}$. Moreover, Wu et al. reported that treatment with live A. muciniphila for 14 days led to the reduced infiltration of neutrophils and macrophages in an immune-mediated liver injury mouse model ${ }^{9}$.

It has been shown that in methionine-choline deficient diet (MCD) mice, steatosis, inflammation, and fibrosis reduce, and the histological futures of the liver tissue improve via induction of insulin-like growth factor (IGF) ${ }^{39}$. Another study showed that use of igf could inactivate the effect of IL- 6 and the inflammatory response in the HepG2 cell line ${ }^{40}$. The results of the present study showed that the mRNA level of igf gene decreased in the PBS group as compared to the healthy control group, while gavage with live and pasteurized $A$. muciniphila and its EVs remarkably restored it in the liver tissue. Interestingly, EVs showed a much better effect on inducing the expression of igf gene compared to the other study groups. Therefore, the igf gene is necessary for insulin sensitivity, with an important role in hormonal and metabolic pathways involved in liver protection ${ }^{41}$. IGF deficiency can cause chronic liver disease (CLD) and primary liver cancer; therefore, an understanding of this condition can provide new insights into the control of liver disease ${ }^{42,43}$. 
Inflammation can be initiated by different pathogen-associated molecular patterns (PAMPs) and damageassociated molecular patterns (DAMPs) through the expression of toll-like receptors (TLRs) in the liver ${ }^{44}$. TLRs are one of the most important members of the pattern recognition receptor (PRR) family, expressed in most types of liver cells. $t l r-2, t l r-4, t l r-5$, and $t l r-9$ genes have been studied more extensively than other types of TLRs in liver diseases ${ }^{45}$. The $t l r-5$ gene activates NF- $\kappa B$ receptors and has been shown to play a major role in many pro-inflammatory responses ${ }^{46}$. Recent studies have reported that the liver is the organ that primarily responds to flagellins by activating the TLR5-MyD88 signaling pathway ${ }^{47,48}$. The role of $t l r-9$ gene in liver disease has been also investigated, and it has been found that $t l r$-9-defective mice are resistant to alcoholic fatty liver disease ${ }^{49}$. Overall, activation of $t l r-9$ gene can lead to liver damage, associated with the increased transmission of bacterial compounds to systemic circulation, a phenomenon observed in many liver diseases ${ }^{50}$.

We previously reported that heat-killed $A$. muciniphila could decrease $t l r-2$ and $t l r-4$ mRNA levels in the LPSactivated LX-2 cell lines and induce HSC regression ${ }^{25}$. In our recent project, we also found that administration of live and pasteurized A. muciniphila and its EVs reduced mRNA levels of different fibrosis markers e.g. $\alpha$-sma, timp, pdgf, tgf- $\beta$ and $t l r-2$ and $t l r-4$ in a similar mouse model ${ }^{51}$. In another study on normal-diet mice also we observed that live and pasteurized $A$. muciniphila reduced the mRNA level of $t \operatorname{lr}-4, \operatorname{tnf}-\alpha$, $\operatorname{tg} f-\beta$ and increased $i l-10$, ppar- $\alpha$ and ppar- $\gamma$ in liver tissue suggesting these bacteria can be considered as new medical supplement to maintain health state and prevent diseases in normal mice ${ }^{52}$. Therefore, in current study we focused on $t \operatorname{tr} 5$ and $t / r 9$ and our results showed that in both quiescent and LPS-activated LX- 2 cells, neither live nor pasteurized A. muciniphila altered the mRNA expression of $t l r-5$ gene, while EVs could ameliorate the expression of $t l r-5$ gene at a concentration of $50 \mu \mathrm{g}$. Treatments could not affect the mRNA level of $t r-9$ gene in quiescent LX-2 cells, while in LPS-activated LX-2 cells, all treatments showed inhibitory effects on tlr-9 genes; the effect of EVs was dose-dependent. In the $\mathrm{HFD} / \mathrm{CCL}_{4}$ mouse model, the use of all three supplementations of $A$. muciniphila decreased the expression of $t l r-5$ and $t l r-9$ genes in the liver tissue.

It has been demonstrated that $t l r-9$ knockout mice with a choline-deficient L-amino acid-defined (CDAA) diet are protected against lipid accumulation, steatohepatitis, and liver fibrosis through suppressing $I L-1 \beta$ production $^{53}$. A recent study by Zhou et al. on $t l r-5$ knockout mice showed that $t r-5$ signaling modulates $\mathrm{CCl}_{4}$-induced liver fibrosis by inducing interferon $\beta$ (IFN- $\beta$ ) expression and regulating $I L-1 \beta$ receptor antagonists (IL-1RA) in mice ${ }^{54}$. In contrast, in another study on tlr-5-deficient mice, the role of $t r$ - 5 in CCl4-induced liver fibrosis was investigated. The results showed that $t l r-5$ was directly involved in the progression of fibrosis by activating the NF- $\mathrm{kB}$ and MAPK signaling pathways. Also, in tlr-5 knockout mice, $\mathrm{CCl}_{4}$-induced hepatic fibrosis was reduced by inhibiting smooth muscle alpha-actin ( $\alpha$-SMA) and collagen expression. Therefore, $t l r-5$ is involved in the progression of fibrosis and may directly or indirectly enhance the development and formation of liver fibrosis by activating HSCs through interactions with other TLR families ${ }^{55}$.

Overall, the findings regarding the exact role of $t l r-5$ in the prevention or induction of liver fibrosis are contradictory, considering the limitations or different models used to induce liver damage. According to the results of the present study and the proven beneficial effects of $A$. muciniphila on the function of the intestinal-epithelial barrier, it can be concluded that the use of A. muciniphila and its derivatives has favorable effects on the liver tissue by improving the intestinal barrier integrity and decreasing the expression of $t l r-5$ and $t l r-9$ genes.

The TLR-dependent HSC stimulation activates Kupffer cells and NF- $\kappa$ B pathways, followed by the production of TGF- $\beta$ and various pro-inflammatory cytokines, such as IL- 6 , IL- $1 \beta$, TNF- $\alpha$, and IL- $8^{56}$. IL- 6 , which can be produced by the liver, is an important determinant of acute-phase response proteins, such as $\mathrm{C}$-reactive proteins (CRP), serum amyloid A (SAA), hepcidin, and other factors originating from the liver tissue ${ }^{57}$. It is clear that IL-6 directly or indirectly stimulates the liver cells and is involved in the development of chronic hepatitis ${ }^{58}$. In contrast, IL-10 is a type II cytokine with anti-inflammatory effects, which plays an essential role in preventing inflammatory and autoimmune damages ${ }^{59}$. Besides, neutralization of IL-10 using antibodies exacerbates hepatic steatosis and insulin resistance in mice ${ }^{60}$. Hepatic and extra-hepatic sources of IL-1 $\beta$, upregulated by activated macrophages, contribute to metabolic liver inflammation steatosis and fibrosis ${ }^{61,62}$.

The results of the present study showed that daily gavage with A. muciniphila and its derivatives not only decreased $i l-6$ gene expression and increased $i l-10$ expression in the liver tissue, but also decreased the tissue levels of these inflammatory and anti-inflammatory cytokines in the liver and adipose tissues of $\mathrm{HFD} / \mathrm{CCL}_{4} \mathrm{mice}$. The mRNA level of $i l-1 \beta$ gene also remarkably decreased in the liver and adipose tissues of mice, gavaged with live and pasteurized A. muciniphila and its EVs. As mentioned earlier, this inhibition of $i l-1 \beta$ was accompanied by a decreased $t l r-9$ mRNA level in the liver tissue.

Moreover, the results of an in vivo study showed the protective effects of a combination of Lactobacillus ( $L$. paracasei and L. casei) and Weissella species on thioacetamide-induced liver injury (TAA). They also found that TAA-treated mice receiving probiotics had significantly less liver damage, which was associated with the reduced liver protein content of TNF- $\alpha^{63}$. Moreover, in vitro and in vivo data from a study by Ashrafian et al. showed that both A. muciniphila and its EVs had stimulatory effects on the expression of $i l-10$ as an anti-inflammatory cytokine. Besides, EVs induced the production of fewer pro-inflammatory cytokines, such as IL-6, IL-8, and IFN- $\gamma$ as compared to the live bacterium ${ }^{37}$.

A recent study by Huck et al. showed that A. muciniphila could counteract the destructive inflammatory effects of Porphyromonas gingivalis in lean and obese animals by inducing the expression of $i l-10$ and reducing pro-inflammatory cytokines ${ }^{64}$. Also, a study by Kang et al. showed that pretreatment with A. muciniphiladerived EVs reduced the production of IL-6 from clone epithelial cells (CT-26), treated with Escherichia coli $\mathrm{EVs}^{62}$. Besides, studies have shown that IL1- $\beta$ plays a key role in adipose and liver inflammation, which is in line with our observations ${ }^{65,66}$. Therefore, induction of IL-10 as an anti-inflammatory cytokine and decreased production of IL-6, TNF- $\alpha$, and ill- $\beta$ as pro-inflammatory cytokines by $A$. muciniphila and its EVs indicate the anti-inflammatory properties of both forms of this bacterium on the liver tissue. In our previous study we also observed that EVs more efficiently reduced the serum level of IL-6, TNF- $\alpha$ and induced IL-10 than other 
groups $^{51}$. This anti-inflammatory effect of EVs can be attributed to the presence of certain compounds in EVs, which regulate immune homeostasis in the intestine, adipose tissue, liver, and other body organs.

PPAR- $\alpha$ and PPAR- $\gamma$ are predominantly involved in reducing inflammatory responses, with protective effects against acute liver injury ${ }^{67}$. PPAR- $\gamma$ plays an anti-fibrotic role by inhibiting downstream TGF- $\beta$ signaling transduction in the liver pro-fibrotic pathways ${ }^{68}$. The PPAR- $\alpha$ pathway has also been shown to be involved in hepatitis and fibrosis and inhibit the activity of inflammatory transcription factors, including NF- $\mathrm{\kappa B}$ and activating protein 1 (AP-1 $)^{69}$. Our results showed that the use of $A$. muciniphila and its EVs increased the expression of both ppar-a and ppar- $\gamma$ genes in the LPS-activated LX-2 cell line. In the HFD/CCL $\mathrm{L}_{4}$ mouse model, a decrease in the expression of these two genes was observed in the PBS group, while in the other treatment groups, a significant increase in the mRNA level of ppar- $\alpha$ and ppar- $\gamma$ genes was observed in both adipose and liver tissues.

In this regard, Choi et al. showed that PPAR- $\gamma$ agonists could prevent TGF- $\beta$-induced fibrogenesis. They found that PPAR- $\gamma$ is a new target in the treatment of liver fibrosis and inflammation ${ }^{70}$. A recent study also showed that administration of Lactobacillus amylovorus for 12 weeks reduced the adipocyte and plasma cholesterol levels and also increased the expression of hepatic and adipose $P P A R-a^{71}$. Consistent with this study, our previous study also showed that $A$. muciniphila and its EVs increased the expression of ppar- $\alpha$ and ppar- $\gamma$ in the adipose tissue of HFD-fed mice and improved fatty acid oxidation and energy metabolism ${ }^{37}$. We have also previously reported that the mRNA level of ppar- $\gamma$ gene was significantly reduced in activated HSCs and interestingly heat-killed $A$. muciniphila could prevent HSCs activation through increasing ppar- $\gamma$ mRNA level in LPS-activated LX- 2 cells ${ }^{25}$. Therefore, since PPAR activation could prevent fatty acid accumulation and regulate inflammatory responses ${ }^{72}$, it can be concluded that $A$. muciniphila and its derivatives might have anti-inflammatory effects on mice with $\mathrm{HFD} / \mathrm{CCL}_{4}$-induced liver fibrosis through induction of PPAR gene expression.

In conclusion, the present results showed that oral administration of A. muciniphila and its derivatives for four weeks could enhance anti-inflammatory responses of the colon, adipose, and liver tissues and subsequently prevent liver injury in $\mathrm{HFD} / \mathrm{CCL}_{4}$ mice. However, we acknowledge several limitations in this study. The main limitation of this study was that we did not determine the content of EVs isolated from A. muciniphila; therefore, we could not explain the exact beneficial mechanism of these bacterial derivatives in the liver function and intestinal hemostasis. Further studies are needed to confirm the effects of A. muciniphila and its derivatives on the prevention of liver injury in humans and explore their potential risks and adverse effects.

Received: 27 July 2021; Accepted: 24 January 2022

Published online: 14 February 2022

\section{References}

1. Seto, W.-K. \& Mandell, M. S. Chronic liver disease: Global perspectives and future challenges to delivering quality health care. Plos one 16, e0243607 (2021)

2. Cotter, T. G. \& Rinella, M. Nonalcoholic fatty liver disease 2020: the state of the disease. Gastroenterology 158, 1851-1864 (2020).

3. Wang, S., Toy, M., Hang Pham, T. T. \& So, S. Causes and trends in liver disease and hepatocellular carcinoma among men and women who received liver transplants in the US, 2010-2019. PloS one 15, e0239393 (2020).

4. Khomich, O., Ivanov, A. V. \& Bartosch, B. Metabolic hallmarks of hepatic stellate cells in liver fibrosis. Cells $\mathbf{9 , 2 4}(\mathbf{2 0 2 0 ) \text { . }}$

5. Kong, M. et al. Ablation of serum response factor in hepatic stellate cells attenuates liver fibrosis. J. Mol. Med. 97, 1521-1533 (2019).

6. Thursby, E. \& Juge, N. Introduction to the human gut microbiota. Biochem. J. 474, 1823-1836 (2017).

7. Jiang, L. \& Schnabl, B. Gut microbiota in liver disease: What do we know and what do we not know?. Physiology 35, 261-274 (2020).

8. Acharya, C. \& Bajaj, J. S. Chronic liver diseases and the microbiome-translating our knowledge of gut microbiota to management of chronic liver disease. Gastroenterology 160, 556-572 (2021).

9. Wu, W. et al. Protective effect of Akkermansia muciniphila against immune-mediated liver injury in a mouse model. Front. Microbiol. 8, 1804 (2017).

10. Derrien, M., Vaughan, E. E., Plugge, C. M. \& de Vos, W. M. Akkermansia muciniphila gen. nov., sp. nov., a human intestinal mucin-degrading bacterium. Int. J. Syst. Evol. Microbiol. 54, 1469-1476 (2004).

11. Reunanen, J. et al. Akkermansia muciniphila adheres to enterocytes and strengthens the integrity of the epithelial cell layer. Appl. Environ. Microbiol. 81, 3655-3662 (2015).

12. Zhang, T., Li, Q., Cheng, L., Buch, H. \& Zhang, F. Akkermansia muciniphila is a promising probiotic. Microb. Biotechnol. 12, 1109-1125 (2019).

13. Chelakkot, C. et al. Akkermansia muciniphila-derived extracellular vesicles influence gut permeability through the regulation of tight junctions. Exp. Mol. Med. 50, e450-e450 (2018).

14. Plovier, H. et al. A purified membrane protein from Akkermansia muciniphila or the pasteurized bacterium improves metabolism in obese and diabetic mice. Nat. Med. 23, 107-113 (2017).

15. Dehghanbanadaki, H. et al. Global scientific output trend for Akkermansia muciniphila research: a bibliometric and scientometric analysis. BMC Med. Inform. Decis. Mak. 20, 1-12 (2020).

16. Roshanravan, N. et al. A comprehensive systematic review of the effectiveness of Akkermansia muciniphila, a member of the gut microbiome, for the management of obesity and associated metabolic disorders. Arch. Physiol. Biochem., 1-11 (2021).

17. Ashrafian, F. et al. Extracellular vesicles and pasteurized cells derived from Akkermansia muciniphila protect against high-fat induced obesity in mice. Microb. Cell Fact. 20, 1-17 (2021).

18. Brown, L., Wolf, J. M., Prados-Rosales, R. \& Casadevall, A. Through the wall: Extracellular vesicles in Gram-positive bacteria, mycobacteria and fungi. Nat. Rev. Microbiol. 13, 620-630 (2015).

19. Raposo, G. \& Stoorvogel, W. Extracellular vesicles: exosomes, microvesicles, and friends. J. Cell Biol. 200, 373-383 (2013).

20. Yáñez-Mó, M. et al. Biological properties of extracellular vesicles and their physiological functions. J. Extracell. Vesic. 4, 27066 (2015).

21. Schwechheimer, C. \& Kuehn, M. J. Outer-membrane vesicles from Gram-negative bacteria: Biogenesis and functions. Nat. Rev. Microbiol. 13, 605-619 (2015).

22. Stentz, R., Carvalho, A. L., Jones, E. J. \& Carding, S. R. Fantastic voyage: The journey of intestinal microbiota-derived microvesicles through the body. Biochem. Soc. Trans. 46, 1021-1027 (2018).

23. Jafari, B., Nejad, R. A. K., Vaziri, F. \& Siadat, S. D. Evaluation of the effects of extracellular vesicles derived from Faecalibacterium prausnitzii on lung cancer cell line. Biologia 74, 889-898 (2019). 
24. Rabiei, N. et al. Induction effects of Faecalibacterium prausnitzii and its extracellular vesicles on toll-like receptor signaling pathway gene expression and cytokine level in human intestinal epithelial cells. Cytokine 121, 154718 (2019).

25. Raftar, S. K. A. et al. The Anti-fibrotic Effects of Heat-Killed Akkermansia muciniphila MucT on Liver Fibrosis Markers and Activation of Hepatic Stellate Cells. Probiot. Antimicrob. Prot., 1-12 (2021).

26. Kang, C.-s. et al. Extracellular vesicles derived from gut microbiota, especially Akkermansia muciniphila, protect the progression of dextran sulfate sodium-induced colitis. PloS one 8, e76520 (2013).

27. Kubota, N. et al. A high-fat diet and multiple administration of carbon tetrachloride induces liver injury and pathological features associated with non-alcoholic steatohepatitis in mice. Clin. Exp. Pharmacol. Physiol. 40, 422-430 (2013).

28. Kim, J. E. et al. Molecular characterization of constipation disease as novel phenotypes in CRISPR-Cas9-generated leptin knockout mice with obesity. Int. J. Mol. Sci. 21, 9464 (2020).

29. Shelby, R. D. et al. Development of a standardized scoring system to assess a murine model of Clostridium difficile colitis. J. Invest. Surg. 33, 887-895 (2020).

30. Chen, X. et al. A mouse model of Clostridium difficile-associated disease. Gastroenterology 135, 1984-1992 (2008).

31. Anzai, Á. et al. Impaired branched-chain amino acid metabolism may underlie the nonalcoholic fatty liver disease-like pathology of neonatal testosterone-treated female rats. Sci. Rep. 7, 1-12 (2017).

32. Kleiner, D. E. Histopathological challenges in suspected drug-induced liver injury. Liver Int. 38, 198-209 (2018).

33. Goodman, Z. D. Grading and staging systems for inflammation and fibrosis in chronic liver diseases. J. Hepatol. 47, 598-607 (2007).

34. Del Campo, J. A., Gallego, P. \& Grande, L. Role of inflammatory response in liver diseases: Therapeutic strategies. World J. Hepatol. 10, 1 (2018)

35. Duarte, S. M., Stefano, J. T. \& Oliveira, C. P. Microbiota and nonalcoholic fatty liver disease/nonalcoholic steatohepatitis (NAFLD/ NASH). Ann. Hepatol. 18, 416-421 (2019).

36. Ilan, Y. Leaky gut and the liver: A role for bacterial translocation in nonalcoholic steatohepatitis. World J. Gastroenterol.: WJG 18, 2609 (2012).

37. Ashrafian, F. et al. Akkermansia muciniphila-derived extracellular vesicles as a mucosal delivery vector for amelioration of obesity in mice. Front. Microbiol. 10, 2155 (2019).

38. Grander, C. et al. Recovery of ethanol-induced Akkermansia muciniphila depletion ameliorates alcoholic liver disease. Gut 67, 891-901 (2018).

39. Nishizawa, H. et al. IGF-I induces senescence of hepatic stellate cells and limits fibrosis in a p53-dependent manner. Sci. Rep. 6, $1-11(2016)$

40. Hribal, M. L. et al. Insulin-like growth factor-I, inflammatory proteins, and fibrosis in subjects with nonalcoholic fatty liver disease. J. Clin. Endocrinol. Metab. 98, E304-E308 (2013).

41. Wheatcroft, S. B. \& Kearney, M. T. IGF-dependent and IGF-independent actions of IGF-binding protein-1 and-2: Implications for metabolic homeostasis. Trends Endocrinol. Metab. 20, 153-162 (2009).

42. Kasprzak, A., Kwasniewski, W., Adamek, A. \& Gozdzicka-Jozefiak, A. Insulin-like growth factor (IGF) axis in cancerogenesis. Mut. Res./Rev. Mut. Res. 772, 78-104 (2017).

43. Takahashi, Y. The role of growth hormone and insulin-like growth factor-I in the liver. Int. J. Mol. Sci. 18, 1447 (2017).

44. Krenkel, O. \& Tacke, F. Liver macrophages in tissue homeostasis and disease. Nat. Rev. Immunol. 17, 306-321 (2017).

45. Cai, J., Zhang, X.-J. \& Li, H. Role of innate immune signaling in non-alcoholic fatty liver disease. Trends Endocrinol. Metab. 29, 712-722 (2018).

46. Nagai, Y., Watanabe, Y. \& Takatsu, K. The TLR family protein RP105/MD-1 complex: A new player in obesity and adipose tissue inflammation. Adipocyte 2, 61-66 (2013).

47. Yan, H. et al. TLR5 activation in hepatocytes alleviates the functional suppression of intrahepatic CD8+ T cells. Immunology 161, 325-344 (2020).

48. Brackett, C. M. et al. Toll-like receptor-5 agonist, entolimod, suppresses metastasis and induces immunity by stimulating an NKdendritic-CD8+ T-cell axis. Proc. Natl. Acad. Sci. 113, E874-E883 (2016).

49. Roh, Y. S., Zhang, B., Loomba, R. \& Seki, E. TLR2 and TLR9 contribute to alcohol-mediated liver injury through induction of CXCL1 and neutrophil infiltration. Am. J. Physiol.-Gastrointest. Liver Physiol. 309, G30-G41 (2015).

50. Bukong, T. N. et al. Abnormal neutrophil traps and impaired efferocytosis contribute to liver injury and sepsis severity after binge alcohol use. J. Hepatol. 69, 1145-1154 (2018).

51. Keshavarz Azizi Raftar, S. et al. The Protective Effects of Live and Pasteurized Akkermansia muciniphila and Its Extracellular Vesicles against HFD/CCl4-Induced Liver Injury. Microbiology Spectrum 9, e00484-00421 (2021).

52. Ashrafian, F. et al. Comparative effects of alive and pasteurized Akkermansia muciniphila on normal diet-fed mice. Sci. Rep. 11, $1-13(2021)$.

53. Miura, K. et al. Toll-like receptor 9 promotes steatohepatitis by induction of interleukin-1 $\beta$ in mice. Gastroenterology 139, 323-334. e327 (2010).

54. Zhou, Z. et al. Toll-Like Receptor 5 Signaling Ameliorates Liver Fibrosis by Inducing Interferon $\beta$-Modulated IL-1 Receptor Antagonist in Mice. Am. J. Pathol. 190, 614-629 (2020).

55. Shu, M., Huang, D.-D., Hung, Z.-A., Hu, X.-R. \& Zhang, S. Inhibition of MAPK and NF- $\kappa B$ signaling pathways alleviate carbon tetrachloride (CCl4)-induced liver fibrosis in Toll-like receptor 5 (TLR5) deficiency mice. Biochem. Biophys. Res. Commun. 471, 233-239 (2016).

56. Kesar, V. \& Odin, J. A. Toll-like receptors and liver disease. Liver Int. 34, 184-196 (2014).

57. Schmidt-Arras, D. \& Rose-John, S. IL-6 pathway in the liver: From physiopathology to therapy. J. Hepatol. 64, 1403-1415 (2016).

58. Widjaja, A. A., Chothani, S. P. \& Cook, S. A. Different roles of interleukin 6 and interleukin 11 in the liver: Implications for therapy. Hum. Vaccin. Immunother. 16, 2357-2362 (2020).

59. Ayelign, B. et al. Association of IL-10 (-1082 A/G) and IL-6 $(-174 \mathrm{G} / \mathrm{C})$ gene polymorphism with type 2 diabetes mellitus in Ethiopia population. BMC Endocr. Disord. 21, 1-8 (2021).

60. Wan, X. et al. PGCla protects against hepatic steatosis and insulin resistance via enhancing IL10-mediated anti-inflammatory response. FASEB J. 34, 10751-10761 (2020).

61. Tilg, H. \& Moschen, A. R. IL-1 cytokine family members and NAFLD: neglected in metabolic liver inflammation. J. Hepatol. 55, 960-962 (2011).

62. Niederreiter, L. \& Tilg, H. Cytokines and fatty liver diseases. Liver Research 2, 14-20 (2018).

63. Jantararussamee, C., Rodniem, S., Taweechotipatr, M., Showpittapornchai, U. \& Pradidarcheep, W. Hepatoprotective effect of probiotic lactic acid bacteria on thioacetamide-induced liver fibrosis in rats. Probiot. Antimicrob. Prot. 13, 40-50 (2021).

64. Huck, O. et al. Akkermansia muciniphila reduces Porphyromonas gingivalis-induced inflammation and periodontal bone destruction. J. Clin. Periodontol. 47, 202-212 (2020).

65. Moschen, A. R. et al. Adipose and liver expression of interleukin (IL)-1 family members in morbid obesity and effects of weight loss. Mol. Med. 17, 840-845 (2011).

66. Nov, O. et al. Interleukin-1 $\beta$ regulates fat-liver crosstalk in obesity by auto-paracrine modulation of adipose tissue inflammation and expandability. PloS one 8, e53626 (2013).

67. Tanaka, N., Aoyama, T., Kimura, S. \& Gonzalez, F. J. Targeting nuclear receptors for the treatment of fatty liver disease. Pharmacol. Ther. 179, 142-157 (2017). 
68. Rudraiah, S., Zhang, X. \& Wang, L. Nuclear receptors as therapeutic targets in liver disease: are we there yet?. Annu. Rev. Pharmacol. Toxicol. 56, 605-626 (2016).

69. Wu, L. et al. Crosstalk between PPARs and gut microbiota in NAFLD. Biomed. Pharmacother. 136, 111255 (2021).

70. Choi, J. H. et al. Platyconic acid A, platycodi radix-derived saponin, suppresses TGF- $\beta 1$-induced activation of hepatic stellate cells via blocking SMAD and activating the PPAR $\gamma$ signaling pathway. Cells 8, 1544 (2019).

71. Nakamura, F. et al. Fragmented lactic Acid bacterial cells activate peroxisome proliferator-activated receptors and ameliorate Dyslipidemia in obese mice. J. Agric. Food Chem. 64, 2549-2559 (2016).

72. Yang, X., Gonzalez, F. J., Huang, M. \& Bi, H. Nuclear receptors and non-alcoholic fatty liver disease: An update. Liver Research 4, $88-93(2020)$.

\section{Acknowledgements}

This article has been extracted from a PhD thesis (Registration No: B-9428) from Mycobacteriology and Pulmonary Research Department, Pasteur Institute of Iran, Tehran, Iran. The study was also funded by a research grant (Project No: RIGLD 1017) from Foodborne and Waterborne Diseases Research Center, Research Institute for Gastroenterology and Liver Diseases, Shahid Beheshti University of Medical Sciences, Tehran, Iran. The authors wish to thank all laboratory staff of the Mycobacteriology and Pulmonary Research Department, Pasteur Institute of Iran, Tehran, Iran; Microbiology Research Center, Pasteur Institute of Iran, Tehran, Iran, and Foodborne and Waterborne Diseases Research Center, Research Institute for Gastroenterology and Liver Diseases, Shahid Beheshti University of Medical Sciences, Tehran, Iran.

\section{Author contributions}

S.K.A. and F.A. designed the project and experiments performed most of experiments, analyzed data and wrote the manuscript. S.A. assisted the laboratory experiments. A.Y. deeply and critically revised the manuscript. H.R.M. dissected, sampled and histologically evaluated the mice. M.M. performed laboratory experiments. S.D. and M.R.Z. supervised the study. S.D, M.R.Z, F.V. and A.M. revised the manuscript. All authors have read and approved the final manuscript version.

\section{Competing interests}

The authors declare no competing interests.

\section{Additional information}

Correspondence and requests for materials should be addressed to S.D.S. or M.R.Z.

Reprints and permissions information is available at www.nature.com/reprints.

Publisher's note Springer Nature remains neutral with regard to jurisdictional claims in published maps and institutional affiliations.

(c) (i) Open Access This article is licensed under a Creative Commons Attribution 4.0 International License, which permits use, sharing, adaptation, distribution and reproduction in any medium or format, as long as you give appropriate credit to the original author(s) and the source, provide a link to the Creative Commons licence, and indicate if changes were made. The images or other third party material in this article are included in the article's Creative Commons licence, unless indicated otherwise in a credit line to the material. If material is not included in the article's Creative Commons licence and your intended use is not permitted by statutory regulation or exceeds the permitted use, you will need to obtain permission directly from the copyright holder. To view a copy of this licence, visit http://creativecommons.org/licenses/by/4.0/.

(C) The Author(s) 2022 